Cahiers « Mondes anciens »

ANCIENS

Histoire et anthropologie des mondes anciens

$2 \mid 2011$

Journées doctorales ANHIMA 2008 et 2009

\title{
Images et mariage, une question de méthode : le geste d'anakalypsis
}

Gaëlle Deschodt

\section{OpenEdition}

Journals

Édition électronique

URL : http://journals.openedition.org/mondesanciens/370

DOI : $10.4000 /$ mondesanciens. 370

ISSN : 2107-0199

Éditeur

UMR 8210 Anthropologie et Histoire des Mondes Antiques

Référence électronique

Gaëlle Deschodt, «Images et mariage, une question de méthode : le geste d'anakalypsis », Cahiers «Mondes anciens » [En ligne], 2 | 2011, mis en ligne le 20 juillet 2011, consulté le 01 mai 2019. URL http://journals.openedition.org/mondesanciens/370 ; DOI : 10.4000/mondesanciens.370

Ce document a été généré automatiquement le 1 mai 2019.

\section{(c) (i) (9)}

Les Cahiers «Mondes Anciens » sont mis à disposition selon les termes de la licence Creative Commons Attribution - Pas d'Utilisation Commerciale - Pas de Modification 4.0 International. 


\title{
Images et mariage, une question de méthode: le geste d'anakalypsis
}

\author{
Gaëlle Deschodt
}

1 Dans les cités grecques, le mariage est un acte social important dans la vie des familles. Il consiste en un rite de passage pour la jeune femme ${ }^{1}$, dramatisant le changement de statut social de la jeune fille en une épouse ${ }^{2}$. Toutefois, les informations sur le mariage sont assez fragmentaires ${ }^{3}$, les sources rares, tardives, et divergentes. Aucune description ne nous est parvenue car les informations sont bien connues des Grecs $^{4}$. Les historiens tentent alors de reconstituer le déroulement à partir des sources littéraires et iconographiques à leur disposition.

2 Cet article traite d'un moment particulier du mariage, assez mal connu: les anakalypteria, et plus particulièrement l'utilisation du geste d'anakalypsis dans les images. Les anakalypteria apparaissent à la fois comme des objets - les cadeaux remis à la mariée ${ }^{5}$ - et comme un geste ou un moment - le dévoilement de la mariée ${ }^{6}$. Ces faits sont à peu près établis; en revanche, le moment et le sens de cette cérémonie font débat parmi les historiens ${ }^{7}$. Florence GHERCHANOC (2006) a consacré un article important aux voiles du mariage, dans lequel elle montre que les polémiques sur le lieu et le moment du dévoilement conduisent à oublier la substance du dévoilement : échange de regards et scellement de l'union. En effet, les mots par lesquels cette étape est également désignée,

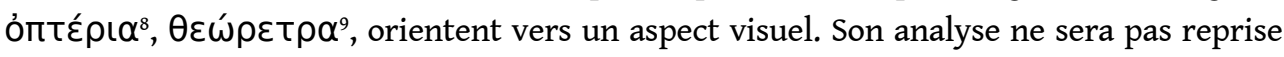
ici ; d'autres historiens ont utilisé les images pour analyser cette étape du mariage, c'est le parti que je choisis. Cela me permettra d'aborder un problème de méthode sur l'utilisation des images et ce que peut signifier ce geste.

3 Les historiens se divisent sur le moment de ce rituel: certains affirment que le dévoilement a lieu à la fin du banquet chez le père de la mariée ${ }^{10}$, d'autres qu'il survient dans la chambre nuptiale ${ }^{11}$, d'autres enfin pensent à un rite double ${ }^{12}$. La céramique est alors mise à contribution pour justifier leur analyse fondée sur des sources littéraires. À chaque position s'attachent des interprétations différentes, qui orientent vers un moment plutôt que vers un autre. 
Deux attitudes se dégagent alors chez les chercheurs dans leur regard sur les images. Certains historiens font une lecture « réaliste » des images. À partir de vases présentant le départ des mariés vers l'oikos de l'époux, ils justifient un dévoilement à la fin du banquet. Par exemple, une amphore de Christchurch, en Nouvelle-Zélande ${ }^{13}$, présente le transfert des époux sur un char, le visage de l'épouse est dégagé, mis en valeur par le contraste du rehaut blanc sur les figures noires. Grâce à ce type de représentation, principalement attique, des chercheurs affirment que le dévoilement a lieu à la fin du banquet chez le père de la mariée car les épouses sont dévoilées sur les vases ${ }^{14}$. Ces remarques montrent une utilisation de l'image comme illustration des textes, une photographie objective de la réalité grecque, ce qu'elle n'est pas.

D'autres historiens sont influencés par les sources littéraires dans leur lecture des images. Il existe très peu de représentations des anakalypteria ${ }^{15}$, d'ailleurs souvent contestées. John OAKLEY (1982) a publié une loutrophore du Peintre de la phiale conservée à Boston ${ }^{16}$ (Fig. 1). Une femme debout tient un voile au-dessus de la tête d'une femme assise. Un Éros apporte une bandelette. À droite, un jeune homme assis et un jeune garçon debout regardent la scène. Au-dessus de la scène, une main amène une grande corbeille contenant des petits objets ronds. Tout à gauche, deux femmes portent divers objets : miroir, boîtes, bandelettes. Le geste correspondrait, selon J. OAKLEY (1982, p. 116-117), aux anakalypteria. En effet, les regards se concentrent sur la jeune mariée, qui baisse les yeux. Les autres femmes apportent les cadeaux reçus par la femme à cette occasion, le panier contient des figues, propices à la fertilité. Les mariées étant non voilées sur les vases, ce rituel prendrait place à la fin du banquet, avant le transfert ${ }^{17}$. C'est une idée préconçue qui oriente l'interprétation.

Fig. 1 : Fragment de loutrophore attique, Boston, Museum of Fine Arts 10.223 ; Peintre de la phiale ; c. 430 ; ARV ${ }^{2}$ 1017.44.

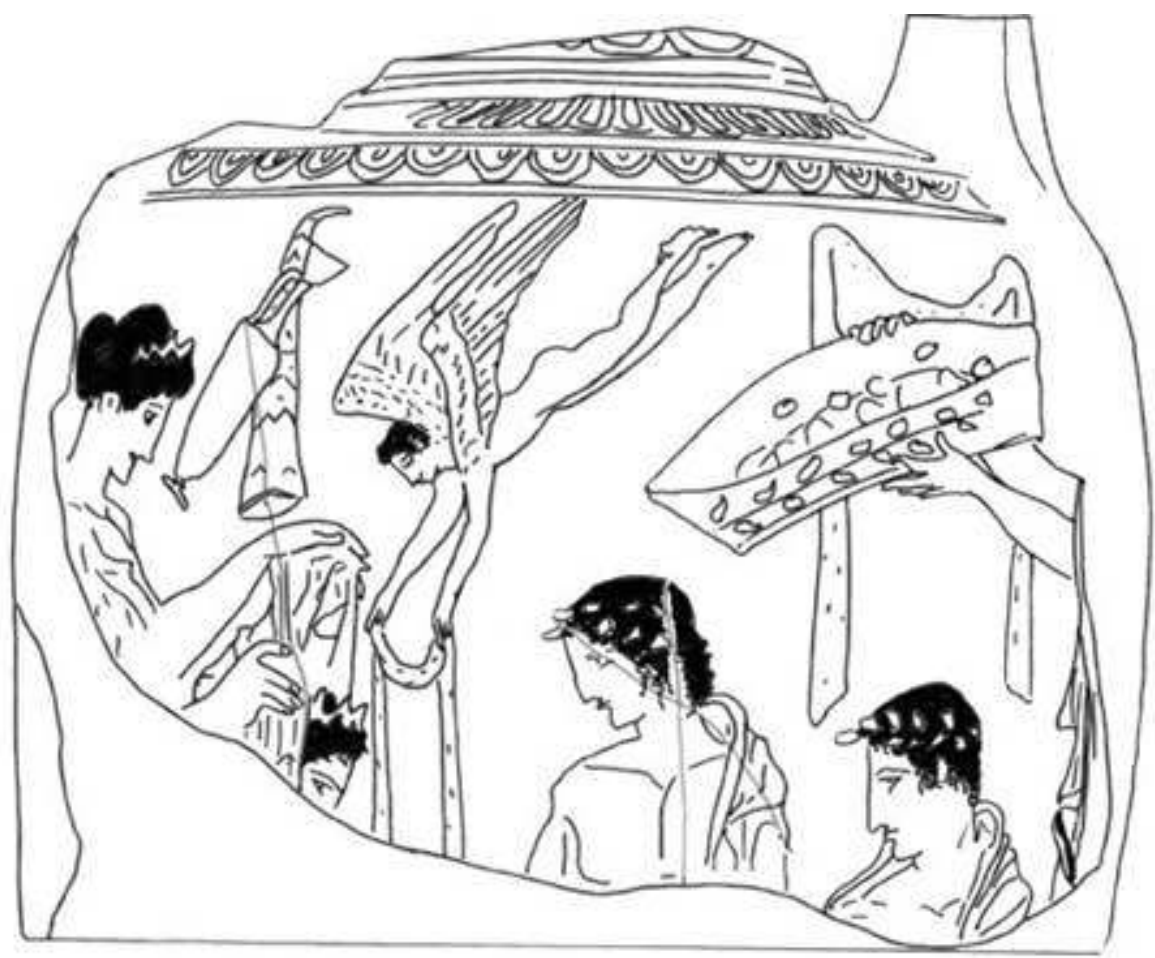


6

Au contraire, Robert SUTTON (1989, p. 353-354) interprète le panier comme un élément du rituel des katakhysmata, rite d'intégration de la jeune épouse à son nouveau foyer ${ }^{18}$, il voit une scène de voilement de l'épouse puisque ces anakalypteria n'ont pas lieu chez l'époux. La mariée est apprêtée pour la chambre nuptiale et la consommation, finalité du mariage, moment au cours duquel elle se dévoile au mari en privé - comme le montrent des terres cuites hellénistiques ${ }^{19}$. Chacun, selon le sens qu'il donne au voile et au dévoilement, selon ses présupposés, interprète la scène différemment.

Or, l'image peinte n'est pas une photographie, ou plutôt un instantané d'un rituel. Elle est une construction à partir de conventions ${ }^{20}$, de moments dont le caractère stéréotypé permet une reconnaissance par le spectateur ${ }^{21}$. C'est John BEAZLEY (1931), qui, le premier, a montré qu'il existe une différence entre ce qui arrive dans la réalité et ce qui mérite d'être représenté dans l'art ${ }^{22}$. Le geste de la nympheutria est précis, elle tient un voile nettement au-dessus de la tête de la mariée. R. SUTTON $(1989$, p. 358) le rapproche du geste très courant sur les vases de réajuster la parure de la mariée comme sur une loutrophore d'Athènes ${ }^{23}$ des années 430 (Fig. 2). L'épouse est emmenée par le poignet par son mari pendant que la nympheutria derrière elle réajuste son manteau sur la tête. Les deux gestes sont différents: le geste du vase de Boston me semble unique. Il s'agit bien d'un dévoilement de la numphè. Par ailleurs, l'analyse du panier comme katakhysmata est très convaincante, le peintre a voulu résumer les temps forts ou importants du mariage pour lui (ou pour son commanditaire) : le dévoilement de la mariée, les katakhysmata, et la parure de la mariée, quel que soit le moment où chacun se déroule. L'image apparaît comme une construction à décrypter.

Fig. 2 : Loutrophore attique, Athènes $1174(\mathrm{CC1224})$; peintre des baigneuses ; c. 430 ; ARV² $1127 / 15$.

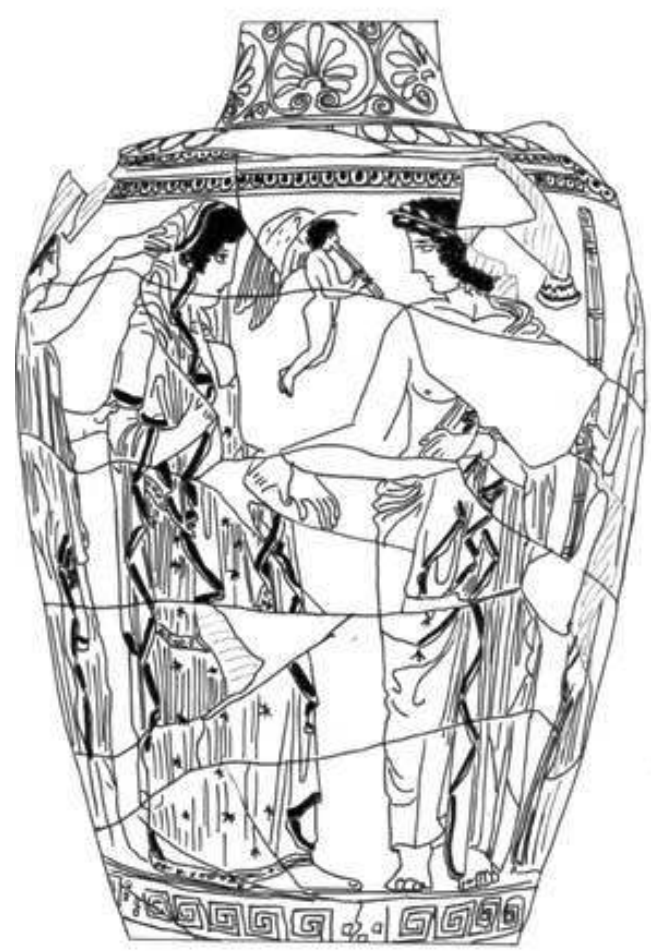

Dessin Gaëlle Deschodt 


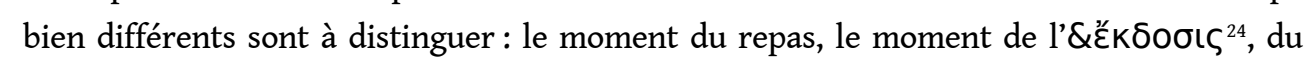
transfert, et le thalamos.

Il n'existe aucune représentation du banquet de noces, c'est un banquet normal qui n'a pas besoin d'être montré sauf lors de déviances, comme ce fut le cas à l'occasion des noces de Pirithoos et d'Hippodamie. Au cours du mariage, des centaures ivres voulurent abuser de la mariée et le banquet se termina en combat rangé25. Le but est alors de rappeler que les usages doivent être respectés sous peine de drames comme l'a montré François LISSARRAGUE (1992, p. 61-62; 1996, p. 430).

De même, le moment intime de la consommation du mariage dans la chambre nuptiale est peu représenté. Les amis chantent et crient pendant qu'un thyroros ${ }^{26}$ garde la porte.

En revanche, le transfert de la mariée vers sa nouvelle demeure en char ou à pied est un moment souvent figuré dans l'iconographie. Les vases montrent quatre types de représentations: le premier correspond à une mariée totalement couverte (Annexe 1) ${ }^{27}$, qui disparait totalement derrière son voile; le deuxième à une épouse qui n'est pas du tout voilée (Annexe 2); le troisième à une épouse tête voilée (Annexe 3), le plus souvent par son manteau ramené en écharpe comme sur une coupe de Berlin ${ }^{28}$ (fig. 3), ou pendant sur ses épaules (Annexe 4), comme sur une loutrophore d'Athènes ${ }^{29}$ (Fig. 2). Enfin, une majorité de représentations montre une mariée avec un voile sur la tête qu'elle écarte (annexe 5) comme sur un lécythe de New York du peintre d'Amasis ${ }^{30}$ (Fig. 4). Le premier et le quatrième type se rencontrent presque exclusivement en figures noires, les autres en figures rouges. Les représentations changent avec le temps, par conséquent, l'interprétation d'une image ne doit pas être faite de façon intemporelle.

Fig. 3 : Coupe attique, Berlin F 2530 ; Peintre d'Amphitrite ; 460-450 ; ARV² 831.20, 1702.

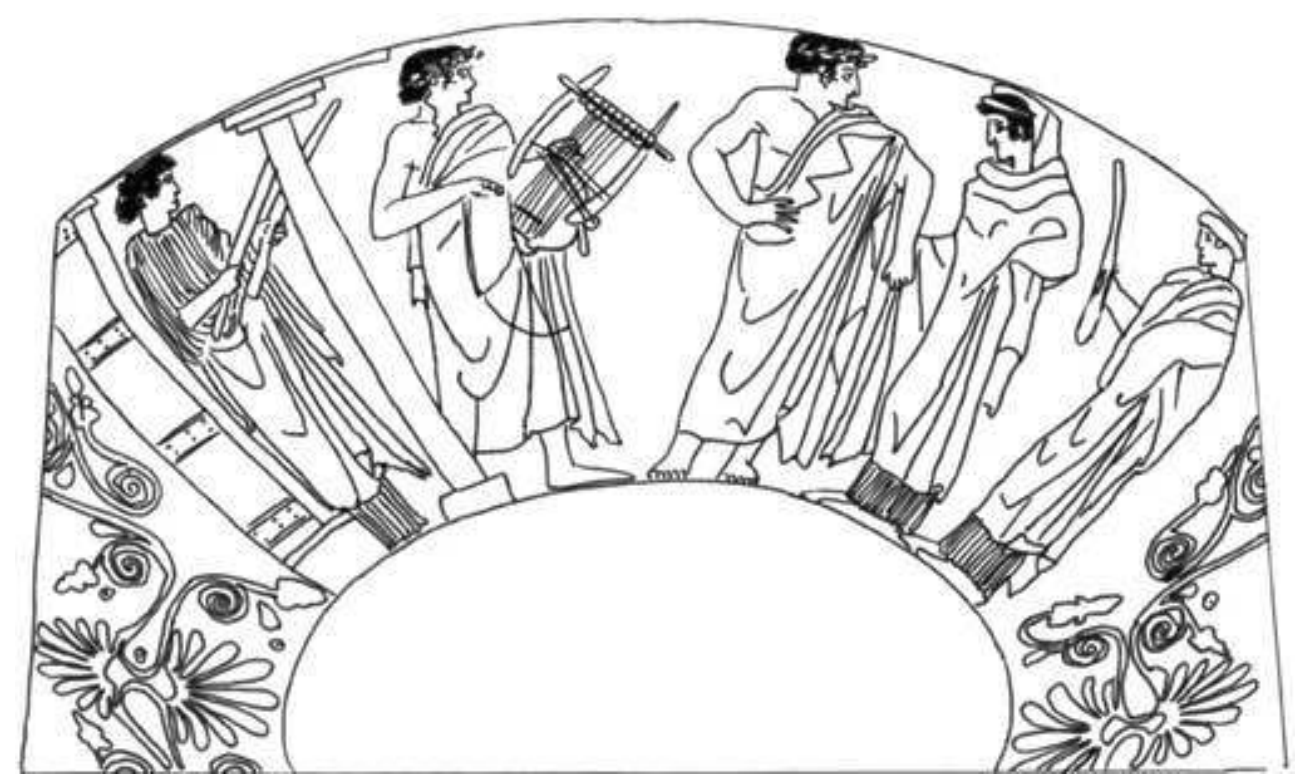

Dessin Gaëlle Deschodt 
Fig. 4 : Lécythe attique, New York, Metropolitan Museum 56.11.1 ; Peintre Amasis ; c. 540.

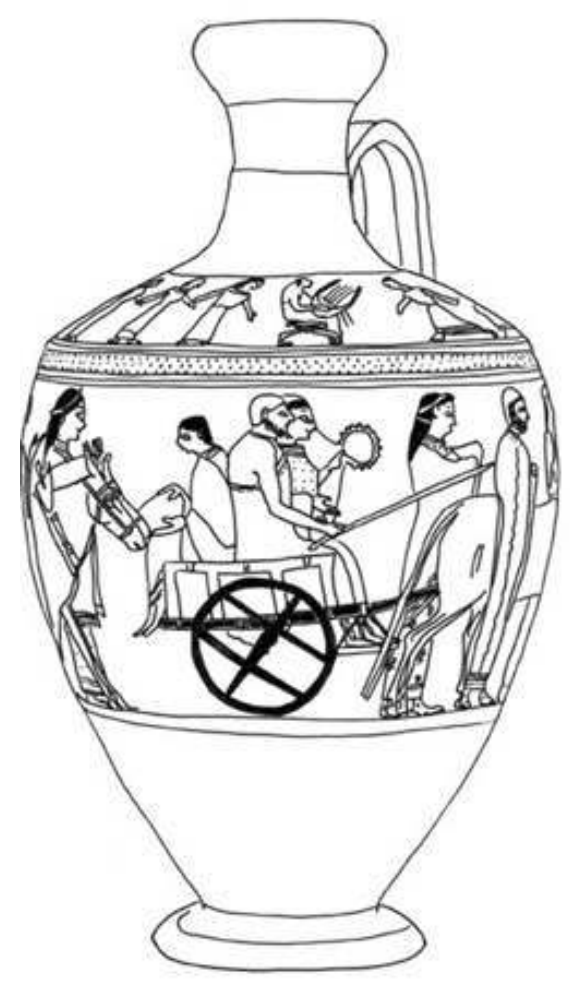

Dessin Gaëlle Deschodt

Le dernier type de représentation correspond au geste appelé anakalypsis. Il existe un désaccord sur son sens ${ }^{31}:$ certains historiens le lisent comme un dévoilement de l'épouse 32 - même si aucun texte ne dit que le bras levé tenant un voile équivaut à dévoiler ${ }^{33}$-, pendant que d'autres l'interprètent comme le fait de cacher le visage ${ }^{34}$; pour d'autres enfin, il s'agit d'un geste plus symbolique que réel indiquant le statut de la femme ${ }^{35}$. Ce motif est généralement interprété comme un geste de mariage par excellence ${ }^{36}$.

Plusieurs divinités accomplissent également ce geste: Amphitrite, Aphrodite, ou Héra ${ }^{37}$. Héra est toutefois la déesse à laquelle ce geste est le plus souvent associée ${ }^{38}$. Sur la frise des Panathénées du Parthénon d'Athènes ${ }^{39}$, elle se retourne vers Zeus, un voile sur la tête tout en l'écartant de la main. Pourtant, la frise du Parthénon ne comporte pas de contexte matrimonial particulier. Cette attitude correspond au dévoilement de la perpétuelle mariée qu'est Héra ${ }^{40}$. Ce geste est assez répandu dans la céramique grecque, tant attique, que des îles ou italiote. Il est caractéristique d'Héra ${ }^{41}$, mais parfois également d'Aphrodite ${ }^{42}$. Ces gestes caractéristiques de certaines déesses sont gratifiants pour les jeunes épouses qui les effectuent. Elles sont à cette occasion assimilées à ces puissances, cela les met en valeur dans ces images intermédiaires entre le réel et l'imaginaire ${ }^{43}$.

Par ailleurs, l'himation sur la tête doit être délicat à porter, il peut glisser sans une main qui le tienne, il permet ainsi de se donner une contenance selon Jean-Marie DENTZER (1982, p. 489). Cette façon de tenir le voile concerne une déesse du mariage, protectrice des femmes mariées ${ }^{44}$, une déesse de la séduction, de l'union charnelle ${ }^{45}$, et des épouses ${ }^{46}$. En fait, peu importe que ce geste soit un geste de voilement ou de dévoilement, il n'est pas un indice pour découvrir quand a lieu la cérémonie des anakalypteria, il s'agit essentiellement de dire un statut social et matrimonial : le nouvel état de femme mariée ${ }^{47}$. 
En effet, le jour du gamos voit la nouvelle épouse quitter le foyer de son père pour passer sous la dépendance de son mari. Au moment du départ de la procession, la kyreia est passée au mari, la femme est donc une épouse, même si le mariage n'est pas encore consommé. Ce geste d'écarter l'himation de la tête exprime visuellement le nouveau statut social de la femme.

Le geste d'écarter le pan de voile se retrouve sur d'autres scènes de mariage. Sur un dinos de Sophilos conservé à Londres ${ }^{48}$, Pélée attend les dieux devant son palais où se trouve Thétis - visible sur le vase François faisant le geste d'anakalypsis ${ }^{49}$. Les dieux arrivent en char et trois déesses écartent leur himation: Héra, Amphitrite, Aphrodite. Elles ne sont pas des nouvelles mariées, mais par ce geste, elles rendent visible leur statut matrimonial. Ce geste n'est pas seulement spécifique de la fiancée lors de son mariage, mais il est présent dans d'autres contextes, comme sur des stèles funéraires ${ }^{50}$, que la femme soit seule $^{51}$, ou qu'elle soit en famille comme sur la stèle de Damasistratè, datée du milieu du $\mathrm{IV}^{\mathrm{e}} \mathrm{s} .{ }^{52}$. La défunte est assise tenant son voile, serrant la main à un homme barbu devant elle. On peut, certes, objecter que ces stèles sont peut-être celles de jeunes mariées, décédées peu de temps après leur mariage, ou de jeunes femmes non encore mariées, à qui l'on fait accomplir ce geste en rappel ou en compensation de la vie qu'elles ne connaitront pas. Cependant, la présence d'une famille empêche d'y porter crédit.

Ce geste est également présent sur des reliefs votifs, en particulier de héros ${ }^{53}$, parfois au banquet $^{54}$. Sur un relief archaïque de Tégée datant du vi ${ }^{\mathrm{e}}$ s., conservé à Sparte ${ }^{55}$, une femme est assise sur un trône et écarte son voile de la main. Un jeune garçon porte une phiale devant une table pour un héros. La femme est l'épouse du héros, ce geste rend visuellement le statut social respectable de la femme mariée.

De même, ce geste se retrouve dans des scènes de départ du guerrier, comme sur une amphore du Louvre ${ }^{56}$ : une femme tend une couronne de la main gauche à un guerrier armé qui part vers la droite, main qui écarte en même temps son himation en geste d' anakalypsis. La scène est encadrée par deux hommes barbus. Elle est typique d'une scène d'armement de guerrier ${ }^{57}$. Ce geste dit de la femme qu'elle est une épouse ${ }^{58}$, celle du guerrier ou celle de son père comme le montrent d'autres vases où Thétis arme son fils Achille, ou bien Hécube arme Hector ${ }^{59}$. Elle tend une couronne à l'hoplite, présage favorable de sa victoire future. Ce geste d'écarter l'himation n'est donc pas spécifique du mariage ou de la fiancée, il dit plus généralement le statut marital de l'épouse.

18 L'utilisation de la céramique pour pallier les sources littéraires est délicate. Les images ont souvent été utilisées comme de simples illustrations des sources écrites. Cette méconnaissance de ce qu'est une image, de la façon dont elle est construite, nuit au raisonnement et à la connaissance des cités grecques. Les images ne se lisent pas en fonction des sources écrites, elles ont leur cohérence et leur fonctionnement propres.

L'utilisation des images comme preuve du moment du dévoilement entraîne des divisions inutiles. Peu importe le moment de ce dévoilement qui a pu varier dans le temps et dans l'espace, le voile et le geste de le tenir sur le côté - ce qui est appelé anakalypsis -, révèlent à eux seuls le nouveau statut matrimonial. Ce geste souligne le passage de l'état de jeune femme à celui d'épouse par un rituel lié à la vue. Le jeu sur les regards est souligné dès les années 430 avant J.-C. par un Éros comme sur un fragment de loutrophore d'Athènes ${ }^{60}$ (Fig. 2).

Les anakalypteria sont un rituel social de dévoilement de la jeune femme qui devient une épouse, mais les images ne sont ni là pour le prouver, ni là pour le décrire : elles sont des 
décors sur des cadeaux offerts à la mariée. Il ne faut pas confondre la cérémonie du dévoilement de la mariée au moment de son mariage avec le geste d'anakalypsis qui dit ce statut dans les images.

\section{BIBLIOGRAPHIE}

\section{Abréviations}

ABV = J. BEAZLEY, Attic black-figure vase-painters, Oxford, 1956.

$A R V^{2}=\mathrm{J}$. BEAZLEY, Attic red-figure vase-painters, Oxford, $2^{\mathrm{e}}$ édition 1963.

Para $=\mathrm{J}$. BEAZLEY, Paralipomena. Additions to Attic black-figure vase-painters and to Attic red-figure vasepainters, Oxford, 1971.

CAT $=$ Chr. W. CLAIRMONT, Classical Attic Tombstones, Kilchberg, 1993-1995.

$C V A=$ Corpus vasorum Antiquorum

LIMC = Lexicon iconographicum mythologiae classicae, Zurich, 1981-1997.

$D A=$ Ch. DAREMBERG, Ed. SAGLIO, E. POTTIER, Dictionnaire des antiquités grecques et romaines, Paris, 1877-1916.

RE $=$ A. PAULY, G. WISSOVA \& al., Real-encyclopädie des Klassischen Altertumswissenschaft, Munich, 1893.

\section{Bibliographie générale}

AVAGianou A. (1991), Sacred Marriage in the Rituals of Greek Religion, Berne.

BEAZLEY J. D. (1931), « Review », JHS 51, 1931, p. 121.

BERGER E. et GISLER-HUWILER M. (2001), Der Parthenon im Basel : Dokumentation zum Fries, Main.

BLUNDELL S. (2001), Women in classical Athens, Bristol, [1998].

BRUit-ZAidman L. (2002), « Les filles de Pandore. Femmes et rituels dans la cité », dans Duby G. et Perrot M. dir., Histoire des femmes en Occident, I. L'Antiquité, Paris, [1991], p. 441-493.

BRULÉ P. (1987), La fille d'Athènes. La religion des filles à Athènes à l'époque classique. Mythes, cultes et société, Paris.

BRULÉ P. (2001), Les femmes grecques à l'époque classique, Paris.

BUXTON R. (1987), « Le voile et le silence dans Alceste », Cahiers du Gita 3, p. 167-178.

CASSIMATIS H. (1988), « À propos de l'utilisation du motif iconographique : autel-trône ? une bizarrerie de l'imagerie », dans Christiansen J. et Melander T. éd., Proceedings of the IIIrd Symposium on Ancient Greek and Related Pottery, Copenhague, p. 117-129.

CAIRNS D. L. (1996a), « "Off with her AI $\Delta \Omega \Sigma$ ” : Herodotus, 1.8.3-4 », ClQ 46, p. 78-83. 


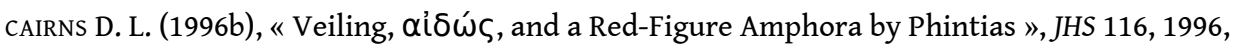
p. $152-158$

CALAME C. (2002), L'Éros dans la Grèce antique, Paris, 1996.

DENTZER J.-M. (1982), Le motif du banquet couché dans le Proche-Orient et le Monde grec du VII au IV siècle avant J.-C., Rome.

DESCHODT G. (2010), « La pudeur, une clé de lecture du mariage grec ancien ? », Hypothèses 2009.

Travaux de l'École doctorale d'histoire de l'université Paris 1, Paris, p. 141-153

DEUBNER L. (1900), « Epaulia », JDAI 15, p. 148-150.

DURAND J.-L. (1979), « Du rituel comme instrumental », dans DETIENNE M. et VERNANT J.-P. éd., La cuisine du sacrifice en Grèce ancienne, Paris, p. 167-181.

ERDMAN W. (1934), Die Ehe im alten Griechenland, New York, 1979.

FERRARI G. (2002), Figures of Speech. Men and Maiden in ancient Greece, Chicago.

FRONTISI-DUCROUX F. et LISSARRAGUE F. (2001), « Corps féminin, corps virginal : images grecques », dans Bruit-Zaidman L., Houbre G., Klapish C. et Schmitt-Pantel P. dir., Le Corps des jeunes filles de l'Antiquité à nos jours, Paris, p. 51-61.

GALT C. M. (1931), « Veiled Ladies », AJA 35, p. 373-393.

GANTZ T. (2004), Mythes de la Grèce archaïque, Paris.

GARLAND R. (1990), The Greek Way of Life from Conception to Old Age, Londres.

GHERCHANOC F. (2006), «Le(s) voile(s) de mariage. Le cas particulier des anakaluptêria », Mètis N.S. 4 , p. 239-267.

GHERCHANOC F. (2009), « Des cadeaux pour nymphai : dôra, anakaluptêria et épaulia », dans Bodiou L. et Mehl V. dir., La religion des femmes en Grèce ancienne, Rennes, p. 207-223.

HAGUE R. (1988), « Marriage Athenian Style », Archaeology 41, n³, p. 32-36.

HALLERAN M. F. (1988), « Text and Ceremony at the Close of Euripides'Alkestis », Eranos 86, p. 123-129.

KAUfFMANN SAMARAS A. (1997), « La beauté d'Héra : de l'iconographie à l'archéologie », dans de La Genière J. éd., Héra, Images, espaces, cultes, Naples, p. 163-171.

KEI N. (2007), « La fleur, signe de grâce dans la céramique attique », Images Re-vues [En ligne], 4, document 8, mis en ligne le 01 janvier 2007, consulté le 27 mai 2011. URL : http:// imagesrevues.revues.org/142

LÉVÊQUE P. et SÉCHAN L. (1990), Les grandes divinités de la Grèce, Paris, [1981].

LISSARRAGUE F. (1990), L'autre guerrier. Archers, peltastes, cavaliers dans l'imagerie attique, Paris.

LisSARRAGUe F. (2002), « Femmes au figuré », dans Duby G. et Perrot M. dir., Histoire des femmes en Occident, I. L'Antiquité, Paris, [1991], p. 203-301.

LISSARRAGUE F. (1992), « Le banquet impossible », dans Aurell M., Dumoulin O. et Thélamon F. éd., La sociabilité à table. Actes du colloque de Rouen, Rouen, p. 54-64.

LISSARRAGUE F. (1995), « Women, boxes, containers : some signs and metaphors », dans Reeder E. dir., Pandora. Women in Classical Greece, Baltimore, p. 91-101. 
LISSARRAGUE F. (1996), « Regards sur le mariage grec », dans Cavalier O. éd., Silence et fureur, Avignon, p. 415-433.

LLEWELLYN-JONES L. (2003), Aphrodite's Tortoise. The veiled Woman of Ancient Greece, Swansea.

MAYo M. E. (1973), « The Gesture of Anakalupteria », AJA 77, p. 220.

MCNIVEN T. (1982), Gestures in Attic Vase Painting : use and meaning, 550-450 B. C., Ph. D. Diss., Université du Michigan.

NEILS J. (2001), The Parthenon Frieze, Cambridge.

NEUMANN G. (1965), Gesten und Gebärden in der griechischen Kunst, Berlin.

OAKLEY J. H. (1982), « The Anakalyteria », AA 97, p. 113-118.

OAKLEY J. H. et SINOS R. H. (1993), The Wedding in Ancient Athens, Madison.

PATTERSON C. (1991), « Marriage and the Married Woman in Athenian Law », dans Pomeroy S. éd., Women's History and Ancient History, Chapel Hill, p. 48-72.

PEMBerton E. G. (1976), « The Gods of the East Frieze of the Parthenon », AJA 80, p. 113-124.

PIRONTI G. (2007), Entre ciel et guerre : figures d'Aphrodite en Grèce ancienne, Liège.

POMEROY S. B. (1997), Families in Classical and Hellenistic Greece. Representations and Realities, Oxford.

REDFIELD J. (1982), « Notes on the Greek Wedding », Arethusa 15, p. 181-201.

REINSBERG C. (1989), Ehe, Hetären und Knaebenliebe im Antiken Griechenland, Munich.

REHM R. (1994), Marriage to death. The Conflation of Wedding and funeral Rituals in greek Tragedy, Princeton.

Roussel P. (1950), « La famille athénienne », Lettre d'humanité, IX, p. 6-59.

SABETAI V. (1997), « Aspects of Nuptial and Genre Imagery in Fifth-Century Athens : Issues of Interpretation and Methodology », dans Oakley J. H., Coulson W. D. E. et Palagia O. éd., Athenian Potters and Painters. The Conference Proceedings, Exeter, p. 319-335.

SABETAI V. (2008), « Women's Ritual Roles in the Circle of Life », dans Kaltsas N. et Shapiro A. dir., Worshipping Women. Ritual and Reality in Classical Athens, New York, 2008, p. 289-297.

SISSA G. (1987), Le corps virginal. La virginité féminine en Grèce ancienne, Paris.

SUTTON R. F. (1981), The interaction between Men and Women Portrayed on Attic Red-Figure Pottery, PhD diss., Université de Caroline du Nord, Chapell Hill.

SUTTON R. F. (1989), « On the Classical Wedding. Two Red-Figured Loutrophoroi in Boston », dans Daidalikon. Studies in Memory of Raymond V. Schoder, S. J., Wauconda, p. 331-359.

TARTAGLiA L. C. (1983), « A Depiction of Veiling in Black Figure Art », AJA 87, p. 264

TOD M. N. et WACE A. J. B. (1906), A catalogue of the Sparta Museum, Oxford.

TOUTAIN J. (1940), « Le rite nuptial de l'anakalypterion », REA LXII, p. 345-353.

VAN GENNEP A. (1981), Rites de passages, Paris, [1909].

VÉRILHAC A.-M. et VIAL C. (1998), Le mariage grec du vi s. a. C. jusqu'à l'époque d'Auguste, Paris.

VERNANT J.-P. (1974), « Le Mariage », PP 38, 1973, repris dans Mythe et Société, Paris, p. 57-81. 
WALTERS H. B. (1898), « On Some Black-Figured Vases Recently Acquired by the British Museum », JHS 18, p. 281-301.

WALTERS H. B. (1921), « Red-Figured Vases Recently Acquired by the British Museum », JHS 41, p. $117-150$.

\section{ANNEXES}

\section{Annexe 1}

Paris, Louvre Cp 11260 ; Munster, Socha XXXX5499 ; Athènes, Agora Museum P19045 + P19584; Munich, Antikensammlungen, 1760 ; Berlin, Staatlichen Museen F 1872 ; New York, Metropolitan Museum 14.105.10 ; Paris, Louvre F 208 ; Paris, Louvre F 232 ; Munich, Antikensammlungen 1406 (J 592) ; Oxford, Ashmolean Museum 1965.108 ; Athènes, musée de l'Agora P 19597a, b, c, d, e ; Athènes, Musée national 1082 ; Athènes, musée de l'Acropole Na 55 Aa 18 ; Bonn, Akademisches Kunstmuseum 994.

\section{Annexe 2}

Épouse non voilée : Paris, Louvre F44 ; Paris, Louvre F 232 ; Athènes, Musée national, 15214 (Acropole 176) ; Paris, Louvre Ca 1961 ; New Haven, Yale University 1913.111 ; Göttingen, Université 23 ; Palerme, Museo V762 (1501 ou 2373 ou 1503) ; Munich, Staatliche Antikensammlungen 2425 ; Athènes, Musée national 1154 ; New York, Metropolitan Museum SL1990.1.21 ; Naples, Museo Archeologico Nazionale (ARV2 532/53) ; Oxford, Ashmolean Museum, 1927.4068 ; Londres, British Museum 1928.5-17.1 ; Toronto, Royal Ontario Museum 635 (929.22.3) ; Thèbes, Musée 31923 ; Berlin, Antikensammlung F2373 ; Athènes, Musée national 1388 (CC1341) ; New York, Metropolitan Museum 75.2.15 ; Budapest, Museum der bildenden Künste T.753.1. Variante tête découverte, mais enveloppée dans son manteau : Naples, Museo Archeologico Nazionale STG36 ; Erlangen, Kunstsammlung der Universität I 301 ; Marathon, Musée K 810 ; Cambridge, Harvard Univ., Arthur M. Sackler Museum 60.353 ; Londres, British Museum 1923.1-18.1 ; Marathon, Musée K329.

\section{Annexe 3}

Coupe attique, Berlin F 2530 ; Naples, Museo Archeologico Nazionale Sant. 36 ; Londres, British Museum B 340 (1843.11-3.83) ; Paris, Petit Palais 309 ; Reading, Public Museum 32.772.1 ; Londres, British Museum D11 ; Florence, Museo Archeologico Etrusco 4025 ; Amsterdam, Allard Pierson Museum 3495 ; Copenhague, Musée National 9080 ; New York, Metropolitan Museum 62.41 ; Jérusalem, Bible Lands Museum 4641 ; Athènes, musée Bénaki 37914 ; Vienne, Kunsthistorisches Museum, 2027 ; Houston, Museum of Fine Arts 37.12 ; Londres, British Museum 1920.12-21.1 ; Bruxelles, Musées Royaux A3547 ; Tampa, Museum of Art 86.78 ; Athènes, Musée national 1630.

\section{Annexe 4}

Naxos, Musée (LIMC Aphrodite 1285) ; Orvieto, Museo Civico XXXX320325 ; Rouen, Musée départemental 447 (9820034) ; Boston, Museum of Fine Art 13.190 ; Berlin, 
Antikensammlung F2205 ; Paris, Louvre G265 ; Boston, Museum of Fine Arts 13.186 ; Tarquinia, Musée national RC 5291 ; Paris, Louvre, G226 ; Athènes, Musée national 1172 (CC 1229) ; Genève, musée d'Art et d'Histoire HR64 ; Boston, Museum of Fine Art 95.44 (P6541) ; Bologne, Museo Civico Archeologico 236 ; Paris, Louvre N3348 (L55) ; Athènes musée Bénaki 35494 ; Wurzburg, Martin von Wagner Museen H4307 (535) ; Munich, Antikensammlungen 9493 ; Londres, British Museum GR 1896.12-17.11; Athènes, Musée national 1174 (CC1224) ; Berlin, Antikensammlung, Staatliche Museen F2372 (collection Sabouroff, inventaire russe AK 942) ; Nauplie, Musée archéologique 309 ; Boston, Museum of Fine Arts 03.802 ; Oxford, Ashmolean Museum 1966.888 ; Londres, British Museum, achat 1910 (WALTERS 1921, p. 143) ; Philadelphia, University of Pennsylvania 5462 ; Tubingen, Eberhard-Karls-Univ. Arch. Inst. E174 (S101279) ; Saint-Pétersbourg, musée de l'Ermitage KAB81A.

\section{Annexe 5}

New York, Metropolitan Museum 56.11.1 ; pinax Berlin (Galt 1931, p. 387, fig. 12) ; Berlin, Staatlichen Museen F 301 ; Athènes, Musée national 354 ; Rome, Villa Giulia (LIMC Héraklès 3331) ; Izmir, Musée archéologique 3332 ; New York, Metropolitan Museum 27.116 ; Los Angeles, County Museum M 77.48.1 ; Christchurch, University of Canterbury, J. Logie Mem. Coll. 43.57 ; Paris, Louvre F205 ; Stuttgart, Marché XXXX350412 ; Athènes, Musée national, collection de l'Acropole 1.1237 ; Saint-Pétersbourg, musée de l'Ermitage B 1403 ; Paris, Louvre CA616 ; Paris, Louvre CP10512 ; Leiden, Rijksmuseum van Oudheden PC44; Athènes, musée de l'Acropole 1957-Aa 423 (NA 969) ; Athènes, musée de l'Acropole 1957-Aa 2589 (NA 279, 981, 1024, 1083) ; Athènes, musée de l'Acropole 1957-Aa 417 (NA 969, 1083) ; Orvieto, Museo Civico 315 ; Melbourne, National Gallery of Victoria 1729.4 ; Naples, Museo Archeologico Nazionale H2498 (81168 ou M979) ; Vatican, Museo Gregoriano Etrusco 358 ; Londres, British Museum B160 ; Munich, Antikensammlungen 1375 (J392) ; Florence, Museo archeologico Etrusco 3799 ; Paris, Louvre F42 ; Vatican, Museo Gregoriano Etrusco 16448 ; Athènes, musée de l'Acropole 1957-Aa 423 (NA 969) ; Londres, British Museum B197 ; Athènes, Musée national 1004 ; Paris, Louvre F 10 ; Athènes, Musée national 12076 ; Tarquinia, Museo Nazionale Tarquiniese RC 1061 ; Paris, Louvre 10651 ; Copenhague, Musée national 113 ; Bâle, Antikenmuseum und Sammlung Ludwig LU25 ; Heidelberg, Université 72/1 ; Jérusalem, Bible Lands Museum 4768 ; Londres, British Museum B174 ; Beverly Hills, Summa Galleries XXXX24294 ; Naples, Museo Nazionale 81308 (H2466) ; Bruxelles, Musées royaux R 310 ; Aberdeen, University 696 ; Copenhague, Musée national B116 (754 ou CHR. VIII754) ; Munich, Antikensammlungen J44 ; Salerne, Museo Civico ; Londres, British Museum B 339 ; Naples, Museo Nazionale Sant. 32 ; Paris, Louvre F 214 ; Toronto, Royal Ontario Museum 919.5.133 ; Athènes, musée de l'Acropole 1957-Aa 305 (NA 268) ; Moscou, Musée Pushkin GMIIM1318 (M1318) ; Pompéi, Museo 19261 ; Boston, Museum of Fine Art 89.562 ; Tübingen, Eberhard-Karls-Univ. H 101205 ; Florence, Museo archeologico Etrusco 94322 ; Florence, Museo archeologico Etrusco 94345 ; Munich, Antikensammlung 1413 (J 693) ; Oxford, Ashmolean Museum 1965.119 ; Florence, Museo archeologico Etrusco 94315 ; Florence, Museo archeologico Etrusco 3790 ; New York, Metropolitan Museum 14.105.10 ; Wurzburg, Universität, Martin von Wagner Mus. L312 ; Copenhagen, Thorvaldsen Museum 74 ; Beverly Hills, Silver XXXX351105 ; San Simeon, State Historical Mon., 5433 (Hearst SSW 9518) ; Munich, Antikensammlung 1406 (J 592); Fiesole, collection A. Costantini XXXX6811; Bruxelles, Musées royaux, R 288 ; Londres, Castle Ashby 20 ; 
Athènes, Musée de l'Acropole, 1957-Aa 187 (NA 969, 1083) ; Athènes, musée de l'Acropole, 1957-Aa 197 (NA 267, 268, 275) ; Athènes, musée de l'Acropole, 1957-Aa 317 (NA 310) ; New York, Metropolitan Museum 98.8.9 ; Rome, Slg. Castellani 22 ; Rome, Villa Giulia 50757 (M437) ; Athènes, collection M. Vlasto XXXX2563 ; Athènes, musée de l'Acropole NA-57Aa-2862; Athènes, musée de l'Acropole NA-55-Aa-1088; Athènes, musée de l'Acropole NA-55-Aa-2843 ; Athènes, musée de l'Acropole NA-57-Aa-1736 ; Athènes, musée de l'Acropole NA-57-Aa-3055.

\section{Annexe 6}

Paris, Louvre F 27 ; Paris, Louvre F 23 ; Munich 1400 (J610) ; Athènes, musée de l'Agora P1227 ; Londres, British Museum sans n(Walters 1898, p. 293, fig. 4) ; Londres, British Museum sans $n^{\circ}$ (Walters 1898, p. 293, pl. 16) ; Paris, Louvre C10656 ; Columbia, University of Missouri, Museum of Art and Archeology 72.22 ; Londres, British Museum 93.7-12.12 ; Paris, Cabinet des Médailles 229 ; Athènes, Musée de l'Agora P1256 ; Toronto, Musée royal 309 ; Gela, Museo archeologico 328 B ; Bruxelles, Musées royaux A910 ; Rome, Villa Giulia 15731.

\section{NOTES}

1. VAN GENNEP 1981, p. 14 ; SUTTON 1989, p. 332 ; OAKLEY et SINOS 1993, p. 3 ; CALAME 1996, p. 130 ; GHERCHANOC 2006, p. 241.

2. BRUIT ZAIDMAN 2002, p. 387 ; GHERCHANOC 2006, p. 241.

3. REINSBERG 1989, p. 49 ; GHERCHANOC 2006, p. 239.

4. LisSarRague 1995 , p. $99 ; 1996$, p. 415.

5. Phérécyde de Syros, frg. 2; Lysias, frg. 331; Harpocration, Souda, Photius, s.v.

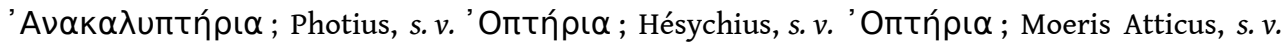
'Oாtńpı $\alpha$; Pollux, II, 59 ; III, 36. Voir GHERCHANOC 2009.

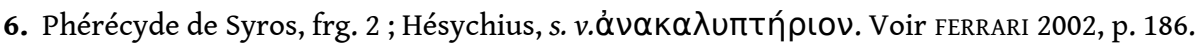

7. FERRARI 2002, p. 186, n. 47.

8. Pollux, II, 59 ; III, 36. Voir Photius, Hésychius, Moeris Atticus, s. v. 'Оп七ńpı $\alpha$; Harpocration, s. v. 'Av $\alpha$ к $\alpha \lambda u \pi \tau n ́ \rho ı$.

9. Harpocration, s. v. 'Av $\alpha \kappa \alpha \lambda \cup \pi \tau n ́ \rho \iota$.

10. DA, s.v. Matrimonium, p. 1650 ; s.v.Anakalypteria", p. 261 ; RE, s.v. Anakalypteria, col. 2031-2032 ; DEUBNER 1900, p. 149 ; ERDMAN 1934, p. 256 ; HALLERAN 1988, p. 127 ; SUTTON 1981, p. 192 ; REDFIELD 1982, p. 192 ; BRULÉ 1987, p. 141-142 ; BRULÉ 2001, p. 187 ; PATTERSON 1991, p. 68, n. 40 ; OAKLEY et SINOS 1993, p. 25 ; CAIRNS 1996a, p. 80 ; FERRARI 2002, p. 187.

11. ROUSSEL 1950, p. 10 ; TOUTAIN 1940, p. 349 ; SISSA 1987, p. 119 ; BUXTON 1987, p. 167 ; HAGUE 1988, p. 35 ; REHM 1994, p. 141-142 ; GARLAND 1990, p. 221 ; VÉRILHAC et VIAL 1998, p. 312.

12. REHM 1994, p. 142 (rite privé devant le mari et rite public associé aux épaulia). LLEWELLYN-JONES 2003, p. 230-238 voit un triple rite.

13. Amphore attique, Christchurch, Université de Canterbury, J. Logie Mem. Coll. 43.57 ; Peintre du deuil du Vatican ; Para, 58/2bis ; CVA Nouvelle Zélande 1, pl. 7, 1-7.

14. DEUBNER 1900, p. 149 ; OAKLEY 1982, p. 113-114 ; SUTTON 1981, p. 165 ; SUTTON 1989, p. 358 ; TOUTAIN 1940, p. 346-347; REINSBERG 1989, p. 59 ; POMEROY 1997, p. 36.

15. FRONTISI DUCROUX et LISSARRAGUE 2001, p. 58 ; LISSARRAGUE 1996, p. 430. 
16. Fragment de loutrophore attique, Boston, Museum of Fine Arts 10.223 ; Peintre de la phiale ; c. 430 ; ARV 1017.44 ; VÉRILHAC et VIAL 1998, pl. 1A ; OAKLEY et SINOS 1993, p. 83, fig. 60-61.

17. OAKLEY 1982, p. 113-114 ; DEUBNER 1900, p. 149 ; TOUTAIN 1940, p. 346-347 ; SUTTON, 1989, p. 358 ; REINSBERG 1989, p. 59.

18. Voir également SABETAI 2008, p. 295-296.

19. SUTTON 1989, p. 358-359. Voir la fig. 2 dans toUtAin 1940 ; LLEWELlyn-JONES 2003, p. 237, fig. 157.

20. FRONTISI DUCROUX et LISSARRAGUe 2001, p. 52 ; CASSIMATIS 1988, p. 117 ; OAKLEY et SINOS 1993, p. 18 ; LISSARRAGUE 1995, p. 99 ; KEI 2007.

21. DURAND 1979, p. 170 ; LISSARRAGUE 1990, p. 35.

22. Voir également SABETAI 1997, p. 321.

23. Loutrophore attique, Athènes 1174 (CC1224) ; Peintre des baigneuses ; c. 430 ; ARV 1127/15 ; OAKLEY et SINOS 1993, fig. 85. Voir également Boston, Museum of Fine Arts 13.186; Munich, Antikensammlungen 9493; Copenhague, Musée national 9080; Paris, Louvre N3348 (L55); Boston, Museum of Fine Arts 03.802 ; Houston, Museum of Fine Arts 37.12; Londres, British Museum 1923.1-18.1.

24. Sur leurs définitions, voir les mises au point de VÉRILHAC et VIAL 1998, p. 230-233 ; VERNANT 1974, p. 58 ; REHM 1994, p. 11-12.

25. GANTZ 2004, p. 496-499. Ces mentions commencent avec Pindare, fr. 166, se développent dans la céramique, ainsi que chez Plutarque, Thésée, 30, 3 et Diodore de Sicile, IV, 70, 3-4.

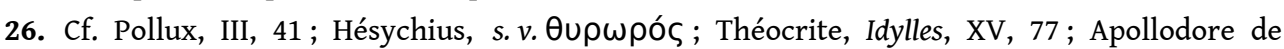
Rhodes, Argonautiques, IV, 1141-1164.

27. C'est une représentation relativement rare, voir OAKLEY et SINOS 1993, p. 31-32.

28. Coupe attique, Berlin F 2530 ; Peintre d'Amphitrite ; 460-450; ARV $231 / 20,1702$; OAKLEY et SINOS 1993, fig. 91 ; CVA Berlin 3, pl. 101.1-4, 106.5-6, 131.2.6.

29. Cf. n. 23.

30. Lécythe attique, New York, Metropolitan Museum 56.11.1; Peintre Amasis ; c. 540 ; OAKLEY et SINOS 1993, fig. 59, 68-70 ; Para, 66. Voir TARTAGLIA 1983, p. 264.

31. Le débat est présenté dans AVAGIANOU 1991, p. 10, n. 51.

32. DEUBNER 1900, p. 149 ; NEUMANN 1965, p. 66 ; OAKLEY 1982, p. 114.

33. LLEWELLYN-JONES 2003, p. 102.

34. TARTAGLIA 1983, p. 264.

35. MAYO 1973, p. 220 ; PEMBERTON 1976, p. 116.

36. LLEWELLYN-JONES 2003, p. 103.

37. Amphitrite : par exemple pinax fragmentaire, Berlin ; époque archaïque ; GALT 1931, p. 387, fig. 12. Aphrodite: par exemple fragment d'amphore mélienne, Berlin, Staatlischen Museen $\mathrm{F}$ 301; deuxième quart du VII ${ }^{\mathrm{e}}$ s.; LIMC Aphrodite 1286, Arès 52. Héra: par exemple amphore attique, Naples, Museo Nazionale 81308 (H2466) ; c. 530 ; proche du Peintre d'Antiménès ; LIMC Héra 275 ; CVA Naples, 1, pl. 7-1 ; CVA Paris, Louvre CA 616 ; c. 570-560 ; ABV 58/122.

38. TARTAGLIA 1983, p. 264.

39. Voir entre autres PEMBERTON 1976, p.115-116, pl. 17, fig. 4 ; BERGER et GISLER-HUWILER 1996 ; NEILS 2001, fig. 124.

40. NEILS 2001, p. 162.

41. Par exemple: rhyton en or, Plovdiv, Musée archéologique 3196; Paestum, Musée 26631; Saint-Pétersbourg, musée de l'Ermitage 16878 ; Naples, Museo Nazionale H 3256 ; Londres, British Museum 770 ; Paestum, Musée (LIMC Héra 442). Voir GALT 1931, p. 384.

42. Par exemple: statuette en terracotta, Paris, Louvre CA 2146; empreinte, Bonn, Akad. Kunstmus. D 697; relief votif, Paris, Louvre MA 742. 
43. KAUFFMANN SAMARAS 1997, p. 164.

44. LÉVÊQUE et SÉCHAN 1990, p. 180-183.

45. Ibid., p. 374-377 ; PIRONTI 2007, p. 11-17, 42-67, 126-135.

46. À la fois épouses mortelles et épouses divines dans le cas des héroïnes, d'Amphitrite notamment.

47. DENTZER 1982, p. 485, 488 ; BLUNDELL 2001, p. 38.

48. Dinos attique, Londres, British Museum 1971.11-1.1 ; Sophilos ; 580-570 ; Para, 19.16bis ; LISSARRAGUE 2002, p. 209, fig. 1 ; LIMC Pélée 211.

49. Cratère à volutes attique, Florence, Museo Archeologico 4209 ; c. 570 ; LIMC Pélée 212.

50. GALT 1931, p. 385 sq.

51. Athènes, Musée national 733.

52. Athènes, Musée national 743 ; CAT 4.430.

53. TOD et WACE 1906, p. 110-112, fig. 1-3, 12.

54. DENTZER 1982, p. 257-261, 484-489.

55. TOD et WACE 1906, p. 110, fig. 12. Voir également LLEWELLYN-JONES 2003, p. 97, fig. 102-103.

56. Amphore attique, Paris, Louvre F 24 ; Peintre Affecté ; 540-520; ARV 247.88; CVA Louvre 3 , pl. 13, 6 et 9. Voir annexe 6. MCNIVEN 1982, p. 102 sq. en fait le catalogue dans son lexique N, geste avec petits objets, dans la section de sens statut marital, sous-partie femmes dans les scènes de départ. Je ne suis pas d'accord avec tous les vases qu'il cite.

57. LISSARRAGUE 1990, p. 44.

58. CAIRNS 1996b, p. 154.

59. Thétis : plat attique, Athènes, Musée national 507 ; ABV 112/56. Hécube : amphore attique, Munich 2307 ; $A R V^{2}$ 26/1.

60. Cf. n. 23. Voir DESCHODT 2010.

\section{RÉSUMÉS}

Les images sont souvent utilisées comme preuve pour déterminer le moment de la cérémonie des anakalypteria selon que la mariée est voilée ou non. Les vases montrent en fait une grande variété de situations. Le geste d'anakalypsis est souvent représenté en figures noires dans le cadre du mariage, mais aussi sur d'autres types de scènes sans connotations matrimoniales. Il ne dit pas s'il est un voilement ou un dévoilement, mais exprime plus vraisemblablement le statut social de la nouvelle épouse.

Images are often used as evidence to determine when the anakalypteria ceremony took place, depending on the fact whether the bride is veiled or not. In fact, vases show a wide variety of situations. The anakalypsis' gesture is often represented in black-figured vases in wedding's scenes, but also in other types of scenes without marriage connotations. That gesture does not say whether it is veiling or unveiling, but it expresses more likely the status of the new bride. 
INDEX

Mots-clés : anakalypsis, mariage, anakalypteria, analyse d'image, voile, dévoilement

Keywords : anakalypsis' gesture, wedding, reading pottery's image, veil, unveiling

\section{AUTEUR}

GAËLLE DESCHODT

Université Paris 1 Panthéon-Sorbonne, ANHIMA - UMR 8210 